\title{
Gilles Siouffi dir. (2020). Une Histoire de la phrase française...
}

Gilles Siouffi, ed. by (2020). A History of the French sentence. From the "Serments de Strasbourg" to digital writing. Arles: Actes Sud, 373 p.

\section{Gérard Vigner}

\section{(2) OpenEdition}

\section{Journals}

Édition électronique

URL : https://journals.openedition.org/dhfles/8624

DOI : $10.4000 /$ dhfles.8624

ISSN : 2221-4038

Éditeur

Société Internationale pour l'Histoire du Français Langue Étrangère ou Seconde

Édition imprimée

Date de publication : 1 décembre 2021

ISSN : 0992-7654

\section{Référence électronique}

Gérard Vigner, « Gilles Siouffi dir. (2020). Une Histoire de la phrase française... », Documents pour

I'histoire du français langue étrangère ou seconde [En ligne], 66-67 | 2021, mis en ligne le 02 février 2022, consulté le 26 mars 2023. URL : http://journals.openedition.org/dhfles/8624 ; DOI : https://doi.org/ $10.4000 /$ dhfles.8624

Ce document a été généré automatiquement le 26 mars 2023.

Tous droits réservés 


\title{
Gilles Siouffi dir. (2020). Une Histoire de la phrase française...
}

\author{
Gilles Siouffi, ed. by (2020). A History of the French sentence. From the "Serments \\ de Strasbourg" to digital writing. Arles: Actes Sud, 373 p.
}

\section{Gérard Vigner}

Gilles Siouffi dir. (2020). Une Histoire de la phrase française. Des "Serments de Strasbourg " aux écritures numériques. Arles : Actes Sud, 373 p.

Enfant chérie des grammairiens, la phrase, quel qu'en soit le projet d'étude, a fait l'objet de très nombreux travaux, qu'il s'agisse de ceux produits par les spécialistes d'histoire de la grammaire, soucieux de cerner les conditions d'émergence et d'évolution d'une notion aux origines relativement récentes, ou de descriptions relevant de ce que l'on peut appeler l'univers des grammaires universitaires ${ }^{1}$ et de celles liées aux préoccupations relevant essentiellement de l'enseignement / apprentissage de la langue. Dans les deux cas, il s'agit de s'intéresser à l'histoire ou à la mise en usage à des fins d'enseignement d'un cadre syntaxique qui, jusqu'à l'orée du XVIII ${ }^{e}$ siècle, ne semblait pas encore disposer d'un statut clairement défini et de faire comme si, dans les périodes antérieures, les locuteurs ou scripteurs avaient pu se dispenser d'en faire usage ${ }^{2}$.

Dans les travaux relevant de l'histoire de la grammaire et portant sur la phrase, on accordera une place toute particulière à l'ouvrage déjà relativement ancien de JeanPierre Seguin, L'invention de la phrase au XVIIIe siècle : contribution à l'histoire du sentiment linguistique français (1993), qui est une histoire de l'émergence et de la stabilisation, encore relative, de la phrase comme nouvelle unité d'analyse de la langue. Sans se livrer à une datation toujours incertaine, notamment sur les origines d'un sens que l'on pourrait qualifier de nouveau, c'est-à-dire anticipant sur les valeurs de sens qui sont celles que nous lui accorderions aujourd'hui, l'étude de Seguin commence par examiner ce qu'il appelle un «sentiment " de la phrase dans le Traité de Grammaire françoise de l'Abbé Reignier-Desmarais de 1705, pour s'achever par l'approche de la phrase dans le premier manuel scolaire que fut la grammaire de Lhomond en 1780. Ce traitement de 
grammairien, est celui que l'on retrouve notamment dans les excellents travaux d'André Chervel, ainsi dans son Histoire de l'Enseignement du français du XVII ${ }^{e}$ au XXe siècle (2006), notamment dans son chapitre consacré à la «Naissance d'une grammaire scolaire du français ", dans laquelle il montre bien encore la confusion qui existe, dans les débuts du XIXe siècle, entre « phrase » et " période».

On peut considérer cependant que la catégorie " phrase » existait déjà dans les usages, avant même que la valeur grammaticale que nous lui connaissons aujourd'hui ne soit installée. De même que Monsieur Jourdain pouvait faire de la prose sans le savoir, de même dans l'histoire de la langue on pouvait faire usage de cet outil d'articulation de la langue, sans disposer d'une définition claire. Ces usages ont une histoire, telle est bien la visée de cet ouvrage. Six grands chapitres, correspondant à six grandes périodes, histoire qui nous conduit des Serments de Strasbourg aux usages contemporains du tweet et du SMS.

Dans le premier chapitre «Du latin tardif au Moyen-Age: les débuts de la phrase française ", Christiane Marchello-Nizia, dans un parcours soigneusement balisé, examine les différents facteurs qui, par le passage du latin à ce que l'on appelle aujourd'hui l'ancien français, ou «rustica romana lingua» pour reprendre la dénomination donnée au concile de Tours en 813, ont permis l'émergence d'une nouvelle organisation de la phrase, distincte de celle des formes de la phrase latine. Deux grands corpus sont examinés, les récits de vies de saints, comme vecteur premier de propagation du français, puis les chansons de gestes, avec pour finir un bref examen des "phrases sans texte ", c'est-à-dire des proverbes. Très tôt interviennent des modifications qui vont donner au français une structure et un rythme différents. Le verbe n'est plus placé à la fin, mais souvent après le sujet, les noms sont introduits par un article, les compléments tendent à se placer après le verbe et le groupe constitué par le sujet, le verbe et l'objet vont se constituer en ensembles difficilement séparables. Et là où en latin les éléments de la phrase sont bien plus autonomes, le français constitue des premiers ensembles, sujet-verbe ou article-nom. Des genres nouveaux, chansons de gestes, romans, en décasyllabes puis en octosyllabes, vont donner naissance à une structuration inédite de la phrase, plus souple car non plus exclusivement psalmodiée. Le développement de la prose permet de mettre en valeur différemment l'information et de jouer avec «l'ordre des mots ». Les conditions de réception du texte vont encore jouer un rôle important, du texte chanté ou simplement oralisé, de la lecture publique ou de la lecture privée, avec à chaque fois une répartition de l'information différente qui va appeler un traitement différencié des propositions. Un examen du «cadre sonore de la langue médiévale ", d'« une langue écrite pour être écoutée ", avec souvent un appel au public, et l'usage nécessaire de procédés de remémoration, confirment cette évolution, avec la généralisation de phrases plus longues. Après avoir étudié la place de "l'auteur revendiqué » à l'opposé de l'anonymat initial, et s'être penchée sur la distinction entre langue écrite et langue orale, Christiane MarchelloNizia montre comment la phrase du français moderne se constitue avant le XIV siècle. Enfin, un bref examen des proverbes montre comment on tire ainsi parti des ressources nouvelles offertes par la structure sujet-verbe-objet ou attribut, ce qui permet de générer des énoncés brefs réutilisables en diverses circonstances.

Bernard Combettes, dans le chapitre suivant «Du moyen français à la Renaissance : phrase et développement de la prose ", inscrit son analyse dans un contexte de large évolution, marqué par de très nombreux changements sociaux, politiques et culturels 
qui vont contribuer à définir et organiser un autre statut du texte écrit. La naissance et le développement de l'imprimerie au milieu du $\mathrm{XV}^{\mathrm{e}}$ siècle ne vont pas manquer d'avoir sur la présentation des textes, mais aussi sur l'organisation des énoncés une influence considérable. Les problèmes que connaît le royaume de France suscitent de nombreux débats et une "communication politique ", donnent lieu à une production intense de libelles, de mémoires, de discours, d'ordonnances, qui font émerger une argumentation en français, appellent une phrase plus complexe, plus riche en outils de subordination et en usage de liens logiques. Dans un monde où tous les intellectuels sont bilingues, le modèle latin va encore jouer un grand rôle et donner à la phrase un profil particulier. Bernard Combettes étudie ainsi l'influence du latin classique, du latin scolastique et du latin de chancellerie, dont l'incidence sur la phrase française sera loin d'être négligeable, accent mis sur les enchaînements logiques, une utilisation constante de la subordination, l'exhaustivité des énumérations, le souci du détail et des circonstances. Du moule latin sort un schéma phrastique nouveau. Dans les nouvelles ou récits de plus grande ampleur des solutions nouvelles émergent, dans l'organisation temporelle, par l'usage des comparaisons, le développement des subordinations et les pratiques de la relance. L'introduction de l'imprimerie donne à la ponctuation une place plus nettement affirmée et fait apparaitre le point-virgule. En poésie le conflit entre décasyllabe et alexandrin, par le choix de la coupe, intervient aussi dans l'organisation de la phrase. L'exemple donné par ceux que Bernard Combettes appelle des « artistes de la phrase ", Christine de Pisant, Jean Calvin et Michel de Montaigne, témoigne de cette richesse et de cette inventivité stylistiques d'un siècle qui accorde au français une place nouvelle. Mais la phrase, au sens moderne du terme, ne constitue pas encore un repère affirmé, tant la période dans son origine rhétorique occupe dans l'univers de l'écriture un repère encore majeur.

7 Gilles Siouffi, dans le chapitre "Entre phrase et période", étudie comment le XVII siècle, par un travail minutieux sur tous les points de la langue, va aider à passer d'une langue encore marquée par de nombreux vestiges d'ancien français à un français "pour ainsi dire moderne». Siècle dans lequel une exigence nouvelle de rigueur, de cohérence dans les choix syntaxiques, de souci de lisibilité, va se faire désormais jour. Ce n'est d'ailleurs pas un hasard si l'Académie française prend naissance en ces tempslà (1634) et que les « remarqueurs », Vaugelas, Bouhours étant les plus connus, ne vont cesser de publier, appelant à une mise en ordre d'une langue dont certains feraient un trop libre usage. Gilles Siouffi va ainsi passer en revue les formes d'écriture, particulièrement variées, d'un siècle riche en productions écrites, en s'interrogeant ainsi sur les choix adoptés et leur incidence sur une certaine conception de l'organisation de la phrase. Après un début consacré à un latin en " paradoxale survie ", selon un ordre des mots qui sent l'influence du français, mais qui aura une certaine influence dans une prose assez latinisante dans les genres didactiques, on plonge d'emblée dans l'examen de la phrase dans la prose narrative, qu'il s'agisse des romans précieux ou des récits de voyages, genres qui constituent une sorte d'espace de liberté, avec notamment de longs enchaînements. Mais les grammairiens sont là qui vont désormais veiller, Malherbe, Vaugelas, Oudin, fondateurs peut-être d'un premier purisme. Tradition rhétorique et idéal de netteté, de simplicité, de clarté vont s'affronter. Gilles Siouffi consacre plusieurs pages fort intéressantes à ce qu'il nomme "la phrase face au vers classique ", relations difficiles on s'en doute. Souvent considéré comme une version noble de la prose, le vers pose plusieurs problèmes. Le vers-phrase est fréquent, par sa superposition entre unité rythmique et unité grammaticale, mais 
avec une forme de régularité qui peut être source d'ennui. Comment alors faire entrer une phrase courte dans un vers et que faire de la phrase longue qui va déborder? Mais s'il y a Malherbe et son goût particulier pour la régularité, on doit compter aussi avec un La Fontaine, plein d'audace qui joue avec les mètres pour loger ainsi des phrases de différentes longueurs. Racine est brièvement approché pour sa capacité à ne pas se laisser enfermer dans "la régularité métronomique de vers faisant phrase ». Suit une brève évocation de Port-Royal et de sa grammaire fondée sur la proposition, dans l'idée qui sera difficile à vérifier, qu'il y a un parallélisme entre la structure de la proposition et sa structure logique. Parcours qui s'achève par un retour sur la phrase oratoire et la phrase écrite, avec Bossuet, l'essor de la correspondance et de la phrase longue et l'âge de la maxime et des formes brèves, avec un panachage entre phrases longues et phrases courtes, que l'on appellera le «style coupé ». Un parcours qui dans son déroulement chronologique ne fait pas apparaitre de choix stylistiques dominants, mais une maitrise progressive, dans des genres variés, d'un outil syntaxique qui pour autant n'est pas encore perçu comme tel, phrase, période, proposition n'étant pas encore clairement distinguées.

Dans le chapitre consacré à la phrase au XVIII siècle, "L'invention de la phrase moderne ", Gilles Siouffi encore note d'emblée que le XVII ${ }^{e}$ siècle livre aux périodes à venir une langue équipée, en dictionnaires, grammaires. Les « remarqueurs » jouèrent en ce domaine un rôle important en produisant des traités de rhétorique divers, pour donner naissance à une langue considérée comme " parfaite ", appelée à rester dans les formes ainsi fixées. Or certains s'inquiètent déjà, dont Fénelon, de ce que l'on pourrait s'enfermer ainsi dans le modèle d'une langue certes plus claire, plus lisible, plus compréhensible intellectuellement, telle que les moralistes en faisaient volontiers usage, mais fort éloignée des traditions de l'éloquence et de l'univers des émotions. Gilles Siouffi va montrer comment, tout au long du XVIII ${ }^{\mathrm{e}}$ siècle, on va faire bouger la langue, et comment la phrase, portée par des exigences stylistiques nouvelles, va connaître des possibilités nouvelles d'évolution. Premier facteur d'évolution, la traduction, non pas d'œuvres issues du patrimoine grec et latin, pratique déjà ancienne, mais venues de l'anglais, langue qui offre un modèle différent de celui du français, langue claire et brève, la traduction de l'anglais devenant au fil du siècle un exercice prisé par de nombreux littérateurs et philosophes. On assiste à l'émergence d'une phrase subjective, où celle-ci va plutôt suivre une réalité faite de circonstances diverses et de sensations, portée par une "écriture à la diable », on écrit vite, avec un goût particulier pour les phrases à rallonge, à l'ossature parfois incertaine, appréciées des mémorialistes et auteurs de lettres. La phrase va bouger car il faut être souvent bref et direct. Mais le rôle des grammairiens se doit d'être particulièrement souligné, ceux grâce auxquels on va passer d'un objet au statut encore incertain, phrase, proposition, période, à celui que le XIX ${ }^{\mathrm{e}}$ siècle va adopter. Gilles Siouffi montre comment les grammairiens vont "inventer la phrase", de Claude Buffier à Charles-François Lhomond en passant par Dumarsais, Nicolas Beauzée ou François-Urbain Domergue. Le terme acquiert un sens et décrit un ensemble verbal appelé à connaitre une longue stabilité. L'auteur poursuit son exploration en montrant comment ce qu'il appelle « la rencontre de voix ", le théâtre, l'opéra, inventant des formes variées qui, quelque part, constituent comme un écho des cultures populaires. Et il faut savoir gré à Gilles Siouffi d'avoir étendu son investigation à ce qu'il appelle « la phrase infinie des peu lettrés ». Usages incertains, souvent éloignés des formes reconnues, mais portés par le besoin de dire, dans l'intensité des émotions ou des doléances vécues ${ }^{3}$. "La phrase enflammée », 
de Rousseau à l'éloquence révolutionnaire, achève ce très riche parcours, dans lequel l'expression de l'émotion, de la passion, qu'elle porte sur l'expression des sentiments ou celle de l'élan révolutionnaire, diversifie formes et usages d'une phrase souvent débordée par l'émotion, même si une certaine tradition classique fondée sur un art de l'éloquence habite encore le discours des révolutionnaires.

Le XIX ${ }^{e}$ siècle s'organise selon un vaste parcours tracé par Jacques Dürrenmatt dont la phrase, telle que la conçoit Chateaubriand, dans la diversité de ses réalisations, sert de point de départ, pour se clore sur le poème-phrase de Mallarmé, Un Coup de dé n'abolira jamais le hasard. Sous l'intitulé La Phrase à l'heure de l'enseignement, Jacques Dürrenmatt marque ainsi une étape nouvelle dans cette histoire. L'enseignement, dans son organisation progressive, dans un appui désormais mieux assuré, définit de nouvelles normes d'organisation, dont l'expression, caricaturale, se retrouve dans la fameuse circulaire qu'aurait adressée Clemenceau aux journalistes de L'Aurore: "Les journalistes ne doivent pas oublier qu'une phrase se compose d'un sujet, d'un verbe et d'un complément. Ceux qui voudront user d'un adjectif passeront me voir dans mon bureau. Ceux qui emploieront un adverbe seront foutus à la porte. » Après une étude de l'écriture de Chateaubriand, Jacques Dürrenmatt étudie le rôle joué par les grammairiens et les éditeurs dans la mise en place de normes qui valorisent un modèle qui se veut simple, les éditeurs reprenant la question de la ponctuation. On aboutit à des solutions variées, le respect du principe grammatical l'emportant sur celui de la restauration de la période. Concurrence du modèle de la phrase longue et de celui de la phrase courte, sur fond de déclin de la rhétorique, historiens et scientifiques hésitant en la matière. L'examen des "phrases non conformes" permet de situer les évolutions qui affectent la phrase sous diverses influences, celles liées au modèle étranger, par le biais des traductions, et par une mise en évidence, non dépourvue d'esthétisation, chez Balzac, Stendhal ou Flaubert, de constructions apparemment déviantes sous forme de déconstructions partielles qui s'efforcent de traduire la spontanéité de l'énonciateur, le caractère non réfléchi de son propos, et ce à des fins comiques ou satiriques. Modèles qui annoncent les usages dans le siècle à venir, phrases apparemment pas ou peu travaillées, incomplètes, ou phrases longues, interminables, avec chez certains un usage délibéré de la non-ponctuation. Rôle joué aussi par les typographes, qui entreront parfois en conflit avec les auteurs, sur l'usage de la virgule ou l'organisation du paragraphe. Le poème de Mallarmé, poème fait pour être vu/lu, plus que pour être oralisé, montre combien la phrase a fait l'objet, par le travail des écrivains, de remaniements considérables, la stabilité de l'objet construit par le travail des grammairiens, permettant alors toutes les audaces.

La phrase au $\mathrm{XX}^{\mathrm{e}}$ siècle est désormais un objet/outil familier à tous, tant par l'abondance des travaux engagés par les grammairiens, qu'il s'agisse des grammairiens universitaires, ou de très nombreux auteurs de grammaires scolaires. L'écrit, qu'il s'agisse de l'écrit littéraire ou de l'écrit des non-lettrés, est constitué désormais en une matière abondante dans laquelle la phrase va connaître les sorts les plus variés. Dans un chapitre intitulé Entre pratiques standardisées et innovations, les deux auteurs, Antoine Gautier et Marie-Albane Watine, entament un très vaste parcours qui les conduit des écritures des " peu-lettrés ", à celles en usage dans les SMS ou sur Twitter, en passant par celles, fort diverses, des écrivains, qu'il s'agisse de Proust, de Céline ou de Claude Simon. La guerre de 14, on le sait, a engendré une correspondance considérable, entre le monde des tranchées et celui des familles. La présence de l'école est remarquée, dans les usages, même si les constructions non normées abondent, avec une influence de 
l'énonciation orale qui en bouleverse les limites. Le rôle de l'école est signalé, dans sa fonction normalisatrice, l'exercice de la rédaction définit un art d'écrire désormais mis à portée du plus grand nombre. Un paradoxe cependant, dans la première Nomenclature grammaticale publiée en 1910, la phrase, concurrencée par la notion de proposition, n'y figure pas. Elle n'est pas encore considérée comme un objet grammatical, mais finira par trouver sa place dans les Instructions de 1938. Mais de fait la phrase est bien là, dans les rédactions, dans les dissertations, inspirée des meilleurs auteurs (voir les travaux d'Antoine Albalat). Antoine Gautier et Amarie-Albane Watine vont alors entreprendre une large revue des usages littéraires qui vont parcourir tout le $\mathrm{XX}^{\mathrm{e}}$ siècle ${ }^{4}$ dans la diversité des sensibilités et des esthétiques associées. Influence aussi des formes de l'oral qui acquièrent une nouvelle légitimité dans la littérature. Raymond Queneau est cité comme il se doit, mais aussi des écrivains qui n'hésitent pas à insérer des tournures qui prétendent de la sorte être fidèles aux différentes formes du langage populaire. Céline est là, notamment pour son «horreur de la phrase bien filée » son goût pour la surponctuation, phrases interrompues. Oralisation bien évidemment stylisée, éloignée des pratiques effectives mais en rupture délibérée avec les usages d'un français académique. La phrase proustienne est aussi longuement analysée, avec ce goût particulier pour les incidentes, les énumérations qui font gonfler la phrase. Forme qui n'a pas été reprise, mais qui montre une époque prête à toutes les réinventions. Et les auteurs de ce chapitre passent ainsi en revue d'autres modèles, phrase courte et averbale, qui connote une expression de la pensée, un dialogue intérieur, chez Valéry Larbaud, Albert Cohen ou Aragon, à cette priorité donnée au continu, sur le modèle proustien, une surintensité qui surgit sans crier gare, sur un autre registre de la subjectivité. Les tenants de la phrase néoclassique ne sont pas oubliés, Julien Gracq ou Marguerite Yourcenar, sans oublier Gide et son fameux « style NRF ». Tout ceci montre à loisir que le $\mathrm{XX}^{\mathrm{e}}$ siècle s'est essayé à toutes les formes, à tous les modèles, sans crainte d'une quelconque norme dominante. L'après-guerre nous offre à nouveaux des phrases longues, mais sousponctuées, plus particulièrement chez Claude Simon. Comme si chaque écrivain s'appropriait un usage sans vouloir s'enfermer dans une école. Cette dernière partie s'achève, au-delà d'une brève analyse de l'incidence des situations de contacts linguistique dans les pays francophones, plus sur le lexique que sur la phrase elle-même d'ailleurs, par la libération de la phrase hors du cadre du texte. Titres, slogans, petites phrases, SMS soumettent la phrase aux traitements les plus variés, les plus inattendus, éloignés des normes d'une phrase scolaire standard, pratiques qui se situent délibérément dans l'exercice d'une nouvelle liberté.

L'ouvrage dans les formes éditoriales adoptées, tout en étant de matière érudite, ne cherche pas pour autant à en écarter le lecteur ordinaire, comme il est souvent d'usage de le faire, par un abondant appareil de notes infra-paginales dont une des fonctions, au-delà de la nécessaire recension des sources, primaires ou secondaires, du savoir mobilisé, est souvent de procéder à une mise à l'écart de celui qui ne partagerait pas le savoir ainsi convoqué. Bien au contraire, les analyses s'enchaînent dans un appui constant aux textes-sources rendant de la sorte la lecture plus aisée, plus continue, reflétant mieux l'évolution des usages qu'il est possible d'éclairer. Une lecture riche en informations variées, parfois trop peut-être, mais il s'agit là d'un travail de stylisticiens, plus que de grammairiens, qui abordent les formes dans leur contexte d'usage et donnent ainsi vie à la langue. 


\section{NOTES}

1. Ainsi de la Grammaire de la phrase française de Pierre Le Goffic, Hachette : 1994.

2. Sans pour autant négliger ce traité des styles que fut l'ouvrage de Gustave Lanson, L'Art de la prose, publié pour la première fois en 1908, qui fit de la phrase le cœur même de son analyse.

3. Arlette Farge, spécialiste du XVIII ${ }^{\mathrm{e}}$ siècle, s'est particulièrement intéressée à cette question, en dépit des difficultés de l'entreprise, dans l'Essai pour une histoire des voix au XVIII siècle, Bayard, 2009.

4. Sur cette question, on se reportera aussi avec intérêt à Gilles Philippe, Sujet, verbe, complément. Le moment grammatical de la littérature française, 1890-1940, Gallimard, 2002, ainsi que Gilles Philippe \& Julien Piat, La langue littéraire. Une histoire de la prose en France de Gustave Flaubert à Claude Simon, Fayard : 2009.

\section{AUTEUR}

\section{GÉRARD VIGNER}

Éducation nationale 